\title{
Active Tension Control for WT Wheelchair Robot by Using a Novel Control Law for Holonomic or Nonholonomic Systems
}

\author{
Jian Wang, ${ }^{1,2}$ Ting Wang, ${ }^{1}$ Chen Yao, ${ }^{1}$ Xiaofan Li, ${ }^{1}$ and Chengdong Wu ${ }^{3}$ \\ ${ }^{1}$ State Key Laboratory of Robotics, Shenyang Institute of Automation, Chinese Academy of Sciences, Shenyang 110016, China \\ ${ }^{2}$ University of Chinese Academy of Sciences, Beijing 100039, China \\ ${ }^{3}$ School of Information Science and Engineering, Northeastern University, Shenyang 110819, China
}

Correspondence should be addressed to Jian Wang; wangjian@sia.cn

Received 8 April 2013; Revised 15 July 2013; Accepted 15 July 2013

Academic Editor: Hamid Reza Karimi

Copyright (C) 2013 Jian Wang et al. This is an open access article distributed under the Creative Commons Attribution License, which permits unrestricted use, distribution, and reproduction in any medium, provided the original work is properly cited.

\begin{abstract}
Interactional characteristics between WT wheelchair robot and stair environment are analyzed, and possible patterns of WT wheelchair robot during the stair-climbing process are summarized, with the criteria of the wheelchair robot for determining the pattern proposed. Aiming at WT wheelchair robot's complicated mechanism with holonomic constraints and combined with the computed torque method, a novel control law that is called active tension control is presented for holonomic or nonholonomic robotic systems, by which the wheelchair robot with a holonomic or nonholonomic mechanism can track the reference input of the constraint forces of holonomic or nonholonomic constraints as well as tracking the reference input of the generalized coordinate of each joint. A stateflow module of Matlab is used to simulate the entire stair-climbing process for WT wheelchair robot. A comparison of output curve with the reference input curve of each joint is made, with the effectiveness of the presented control law verified.
\end{abstract}

\section{Introduction}

Wheelchair robot, as a very important service mobile robot for some aged and physically disabled persons, is attracted by more and more domestic and foreign research institutions in the recent decades. The focus of the study, however, is mainly on the adoption and development of traditional obstacle-navigation mechanisms to realize the stair-climbing performance. Such mature obstacle-navigation mechanisms applied to the wheelchair robot as wheel mechanism [1], leg type mechanism $[2,3]$, crawler mechanism [4], and their composite mechanism [5] have their own advantages and disadvantages, respectively. This paper employs the WT wheelchair robot developed by Ting Wang in the Chinese Academy of Sciences, Shenyang Institute of Automation, as the experimental platform, which uses a deformable wheeltracked obstacle-navigation mechanism and also introduces complicated holonomic constraints.

Control problems for holonomic and nonholonomic robot systems have been initiated since the 1990s of last century. Then, there is a large amount of published literature that proposed various control laws to solve such problems [6], most of which are combined with dynamic models expressed in the d'Alembert-Lagrange form [7].

From the existing literature, control problems for holonomic and nonholonomic robot systems are developed parallel with different approaches, respectively, with no unified theoretical framework proposed to design a controller applicable to both of them.

For holonomic robot systems, McClamroch and Wang [8], who make a pioneering work, present a feedback control law that can follow the reference input generalized coordinates and the reference input constrained forces of holonomic constraints at the same time for the motion/force controller design of holonomic constrained robot systems. But unfortunately they do not figure out the exact relationship between generalized coordinates and constrained forces in the derivation process, with a force-tracking residual error appearing in the proposed control law. Then follow the adaptive control [9] that has assured force tracking errors to be zero, the robust control [10] that deals with unstructured uncertainties, and the intelligent control [11-13] that 
deals with nonlinearity. However, there is always a problem common to them which is that they are unable to remove the force-tracking residual error in their control laws in principle.

On control problems for nonholonomic robot systems, Bloch [14] makes an earlier work. And many other control methods for such problems such as adaptive control [15], robust control [16], and intelligent control [17-19] are proposed subsequently. However, there is also a defect common to them that in their selected dynamic model $\mathbf{D} \ddot{\mathbf{q}}+\mathbf{C} \dot{\mathbf{q}}+\mathbf{G}=$ $\mathbf{B} \boldsymbol{\tau}+\mathbf{A}^{T} \boldsymbol{\lambda}$ (where $\mathbf{D} \in \mathbb{R}^{n \times n}$ is the inertia matrix, $\mathbf{C} \in \mathbb{R}^{n \times n}$ is the centripetal and Coriolis matrix, $\mathbf{G} \in \mathbb{R}^{n}$ is the gravitation force vector, $\mathbf{B} \in \mathbb{R}^{n \times r}$ is the input transformation matrix, $\boldsymbol{\tau} \in \mathbb{R}^{r}$ is the input vector, $\mathbf{A} \in \mathbb{R}^{m \times n}$ is the vector associated with the constraints with $r \geq n-m$, and $\lambda \in \mathbb{R}^{m}$ is a Lagrange multiplier presenting the constrained force), the introduction of the so-called input transformation matrix $\mathbf{B}$ makes the dynamics well as equivalent to $\mathbf{D} \ddot{\mathbf{q}}+\mathbf{C}+\mathbf{G}=\left[\begin{array}{c}\mathbf{0} \\ \boldsymbol{\tau}\end{array}\right]+\mathbf{A}^{T} \boldsymbol{\lambda}$ so that the controller $\left[\begin{array}{l}\mathbf{0} \\ \boldsymbol{\tau}\end{array}\right]$ is able to control only $r$ variables, as giving up the control of the remaining $n-r$ variables, such as some of the constrained forces of the nonholonomic constraints.

In this paper, first, the interactional characteristics between the mobile wheel-tracked mechanism and stairs are analyzed and summarized, with possible patterns and criteria of WT wheelchair robot during the stair-climbing process proposed. Finally aiming at the need for controlling tension forces of the tracks, namely, the constraint forces of the holonomic constraints, for WT wheelchair robot, a control law applied to holonomic system or nonholonomic system is derived from the general form of the Hamilton principle [20] and the deduction of Lagrange's equations with the Lagrange multipliers. And if some proper treatments are handled, such theoretical framework can be applied to the control problem for nonholonomic robot systems also.

\section{Robot Description}

2.1. Experiment Platform of WT Wheelchair Robot. WT wheelchair robot, as shown in Figures 1(a) and 1(b), is mainly composed of a body, two front flippers, two driving wheels, a back flipper, and two tracks installed symmetrically. The body $A B$ contains a supporting frame and a seat connected together, with the guide wheels $A$, the carrier wheels $B$, and the belt pressing wheels $F$ and $G$ symmetrically arranged at both sides of the supporting frame. The back flipper $B E$ can be controlled by a motor which can rotate around the axis $B$ of the supporting frame. The driving wheels $E$ and $E^{\prime}$, which equip walking motors to control walking, are arranged symmetrically at the tip of the back flipper. The left front flipper $C D$ and the right front flipper $C^{\prime} D^{\prime}$ are controlled by motors, respectively, which can rotate around the axis $C C^{\prime}$ of the supporting frame to adjust the track tension, respectively, and there are the belt pressing wheels $D$ and $D^{\prime}$ arranged at the tip of each front flipper, respectively. There are the same two tracks arranged at both sides of WT wheelchair robot. So WT wheelchair robot has 5 driving motors, with a potentiometric sensor installed in the axis $B$ for detecting the swing angle of the back flipper $B E$ and a two-dimensional tilt sensor for detecting changes of the pitch angle and the roll angle.
2.2. Stair-Climbing Process. Stair-climbing process can be defined as the one that WT wheelchair robot transfers from the horizontal ground to the slope of stairs. Experimental results show that the process is so short that the influence of turning direction over the motion is negligible and that the process can be simplified on the vertical plane.

As is shown in Figure 1(c), the stair-climbing process of WT wheelchair robot can be described as follows. First, rotate the back flipper counterclockwise so that the tension forces of the tracks are increased, and then rotate the front flippers clockwise or counterclockwise so that the track tensions remain constant. Finally, the plane angle of the seat corresponding to the horizontal plane can be controlled by swinging the back flipper to different angles and driving the wheelchair robot backwards.

Shown in Figure 1(b) is the relative coordinate frame, whose origin is located at the axis of the wheel $A$ with its $u$ axis parallel to the plane of the wheelchair seat and its $v$ axis perpendicular to the plane of the wheelchair seat. $q_{1}$ can be defined as the angle rotated counterclockwise from $B A$ to $B E . q_{2}$ and $q_{3}$ can be defined as the angles of the left and right front flippers rotated clockwise from $C A$ to $C D$ and to $C^{\prime} D^{\prime}$, respectively. $q_{4}$ and $q_{5}$ can be defined as the angles of the left and right driving wheels rotated clockwise around the back flipper BE, respectively. The variables defined above can be regarded as the generalized coordinates of WT wheelchair robot. $q_{6}$ and $q_{7}$ can also be defined as the angles measured by the two-dimensional tilt sensor installed on WT wheelchair robot. The rest are structural parameters that can be measured, see Table 1 .

Perimeters of both tracks are equal to each other and remain unchanged, namely, $L=L^{\prime}$, so that the two holonomic constraint equations of the robot can be obtained as follows:

$$
\begin{aligned}
f\left(q_{1}, q_{2}\right)= & r_{A} \angle A+L_{A D}+r_{D} \angle D+L_{D F}+r_{F} \angle F+L_{F B} \\
& +r_{B} \angle B+L_{B E}+r_{E} \angle E+L_{A E}-L=0, \\
f\left(q_{1}, q_{3}\right)= & r_{A} \angle A+L_{A D^{\prime}}+r_{D^{\prime}} \angle D^{\prime}+L_{D^{\prime} F}+r_{F} \angle F \\
& +L_{F B}+r_{B} \angle B+L_{B E^{\prime}}+r_{E}^{\prime} \angle E^{\prime}+L_{A E^{\prime}}-L^{\prime}=0,
\end{aligned}
$$

where $r_{I}$ is radius of the wheel $I, \angle I$ is the central angle corresponding to the track around the wheel $I$, and $L_{I J}$ is the tangent length between the wheels $I$ and $J$ which can be expressed as the function of $q_{1}$ and $q_{2}$ or that of $q_{1}$ and $q_{3}$.

\section{Analysis and Determination of Patterns of WT Wheelchair Robot during Stair-Climbing Process}

By pattern in this paper meant the different situations in which the wheelchair robot has different interactions with stairs during the stair-climbing process and has different dynamics. 


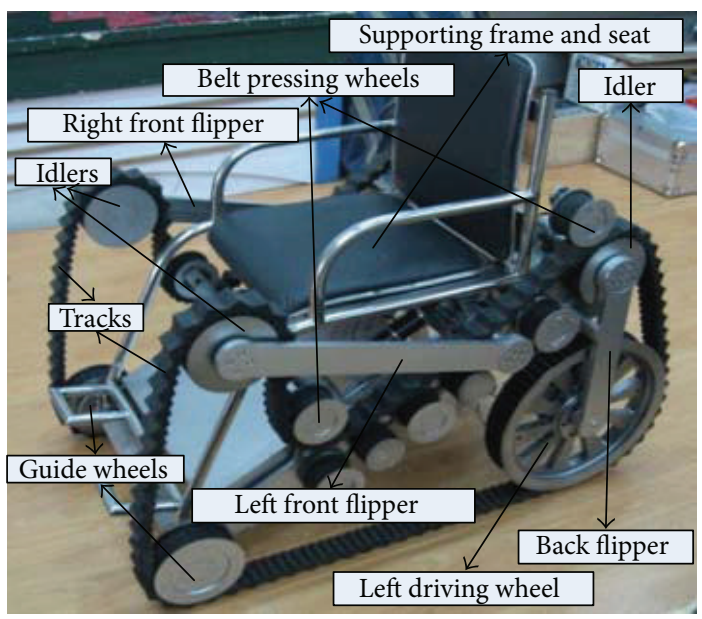

(a) Experimental platform of WT wheelchair robot

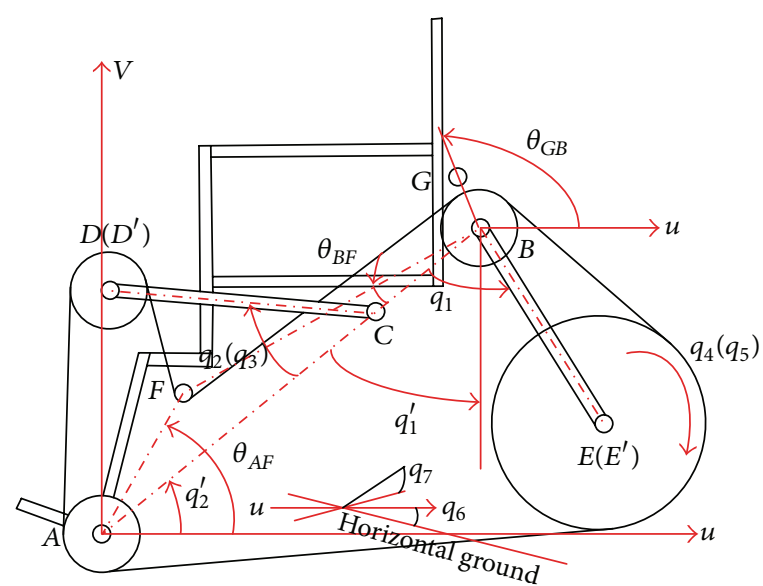

(b) Generalized coordinates of WT wheelchair robot

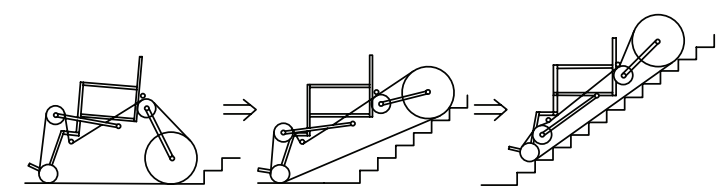

(c) Stair-climbing process of WT wheelchair robot

FIGURE 1: WT wheelchair robot.

TABLE 1: Parameters of WT Wheelchair Robot.

\begin{tabular}{lccccccc}
\hline & \multicolumn{3}{c}{ Structure $(\mathrm{m})$} & \multicolumn{2}{c}{ Mass $(\mathrm{kg})$} & \multicolumn{2}{c}{ Inertia $\left(\mathrm{kg} \cdot \mathrm{m}^{2}\right)$} \\
\hline$l_{B E}$ & 0.37 & $r_{E}$ & 0.2 & $m_{E}$ & 15 & $J_{E}$ & 0.3 \\
$l_{A B}$ & 0.94 & $r_{A}, r_{B}, r_{D}$ & 0.07 & $m_{B E}$ & 2.5 & $J_{B E}$ & 0.03 \\
$l_{C D}$ & 0.53 & $r_{F}, r_{G}$ & 0.04 & $m_{A B}$ & 55 & $J_{A B}$ & $J_{C D}$ \\
$l_{A F}$ & 0.31 & $L$ & 3.65 & $m_{C D}$ & 3 & 0.04 \\
$l_{A C}$ & 0.69 & & & & & \\
\hline
\end{tabular}

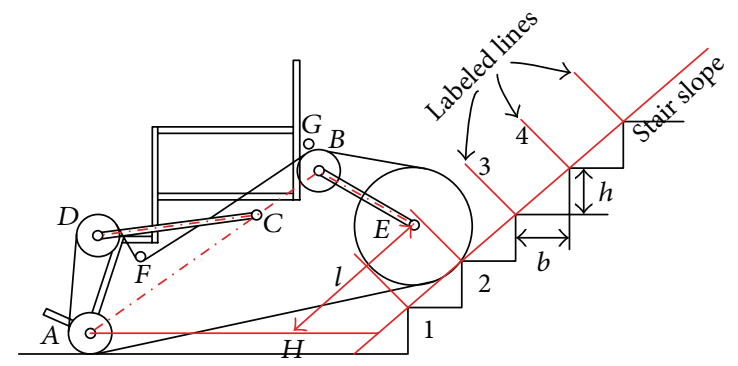

FIGURE 2: Sketch map of demarcation of stairs.

3.1. Location of the Wheelchair Robot on Stairs. The height of a single stair is $h=0.15(\mathrm{~m})$, and the width is $b=0.32(\mathrm{~m})$, with slope angle of stairs defined as $\Lambda=\arctan (h / b)$. For connecting all stair vertices, one can get a stair slope line, as shown in Figure 2. One can draw straight lines, which are called labeled lines, through stair vertices perpendicular to stair slope, on the basis of which he or she can number stairs from bottom to top in turns. When the center of the driving wheel is located between the two certain straight lines, one can denote the robot by using the smaller number of the two.
An entire stair-climbing process can be decomposed into a number of segments, from the center of the wheel $E$ running after a labeled line to that running after the next one. Also one can define $l=|E H|$ as a parameter to calculate the line number labeled for the robot, where $|E H|$ is the length of the line $E H$ with $H$ being the point crossed by the line $E H$ which is parallel to the stair slope and the line $A H$ which is parallel to the horizontal ground.

Criterion expressed by $l$ for labeling the robot or stair can be described as:

$$
\begin{aligned}
& i \sqrt{h^{2}+b^{2}}+r_{E} \frac{b}{h}-\frac{r_{A} \sqrt{h^{2}+b^{2}}}{h} \\
& \quad \leq l<(i+1) \sqrt{h^{2}+b^{2}}+r_{E} \frac{b}{h}-\frac{r_{A} \sqrt{h^{2}+b^{2}}}{h} .
\end{aligned}
$$

Thus, the line number labeled for the robot or stair can be deducted as follows:

$$
i=\text { floor }\left(\frac{h l+r_{A} \sqrt{h^{2}+b^{2}}-r_{E} b}{h \sqrt{h^{2}+b^{2}}}\right),
$$

where floor $(\bullet)$ is a rounding function. 


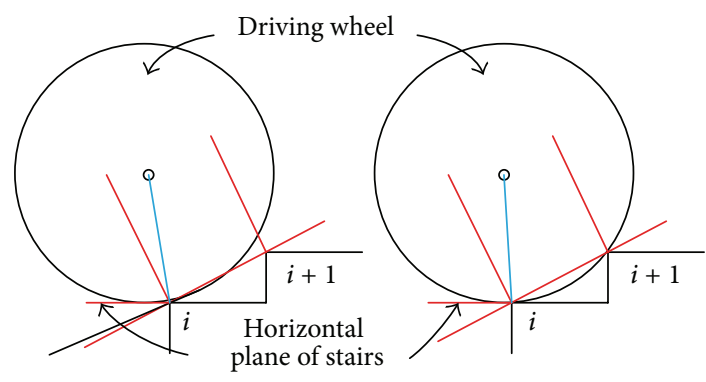

FIGURE 3: Primary classification of patterns.

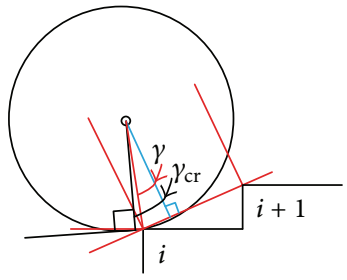

Pattern I

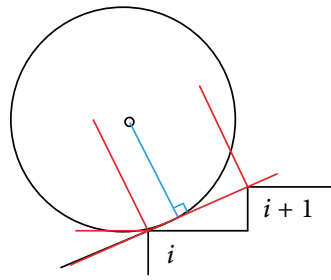

Pattern II
Figure 4: Classification between Pattern I and Pattern II.

3.2. Pattern Classification. The situation that horizontal surface of stairs is tangential to the bottom surface of the driving wheel, as shown in Figure 3, can be used as a primary classification of the patterns of the robot. When the driving wheels move on the horizontal plane, as shown in Figure 3, the parameter $L$ remains unchanged, and the relationship can be obtained as follows:

$$
i \sqrt{h^{2}+b^{2}}+\frac{\left(r_{E}-r_{A}\right) \sqrt{h^{2}+b^{2}}}{h}=l .
$$

3.2.1. Classification between Pattern I and Pattern II. When $i \sqrt{h^{2}+b^{2}}+\left(r_{E}-r_{A}\right) \sqrt{h^{2}+b^{2}} / h>l$, there are two patterns, as shown in Figure 4.

Pattern I. The stair vertex with the smaller labeled line number i, as shown in the left subfigure of Figure 4, applies a force to the track and then to the driving wheels. The criterion for determining Pattern I can be described as follows:

$$
\begin{gathered}
i \sqrt{h^{2}+b^{2}}+\frac{\left(r_{E}-r_{A}\right) \sqrt{h^{2}+b^{2}}}{h}>l, \\
\gamma \leq \gamma_{\mathrm{cr}},
\end{gathered}
$$

where $\gamma$ is the angle formed by the line crossing through from stair vertex to the center of the driving wheel and the line perpendicular to stair slope and $\gamma_{\mathrm{cr}}$ is the angle formed by the line crossing through from the tangent point between track and driving wheels to the center of the driving wheel and the line perpendicular to stair slope.

Pattern II. The stair vertex with the smaller labeled line number $i$, shown as the right subfigure of Figure 4, applies a force to the track and not to the driving wheels directly.

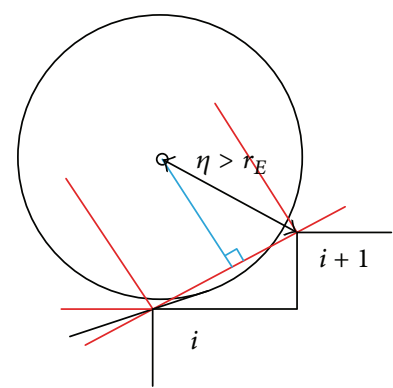

Pattern III

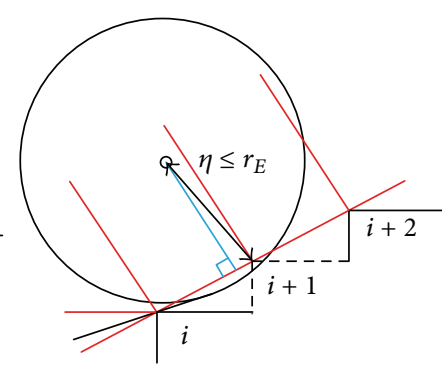

Pattern IV
Figure 5: Classification between Pattern III and Pattern IV.

The criterion for determining Pattern II can be described as follows:

$$
i \sqrt{h^{2}+b^{2}}+\frac{\left(r_{E}-r_{A}\right) \sqrt{h^{2}+b^{2}}}{h}>l,
$$

$$
\gamma>\gamma_{\mathrm{cr}}
$$

3.2.2. Classification between Pattern III and Pattern IV. When $i \sqrt{h^{2}+b^{2}}+\left(r_{E}-r_{A}\right) \sqrt{h^{2}+b^{2}} / h \geq l$, there are also two patterns, as shown in Figure 5.

Pattern III. The stair vertex with the larger labeled line number $i+1$, shown as the left subfigure of Figure 5, remains in no contact with tracks, thus applying no force to the driving wheels directly. While the stair vertex with the smaller labeled line number $i$, shown as the left subfigure of Figure 5, applies a force to tracks and not to the driving wheels directly. The criterion for determining Pattern III can be described as follows:

$$
\begin{gathered}
i \sqrt{h^{2}+b^{2}}+\frac{\left(r_{E}-r_{A}\right) \sqrt{h^{2}+b^{2}}}{h} \leq l, \\
\eta>r_{E}, \quad \zeta<\frac{\pi}{2},
\end{gathered}
$$

where $\eta$ is the distance from the center of the driving wheels to the stair vertex with the larger labeled line number and $\zeta$ is the angle formed by stair slope and the line crossing from the center of the driving wheels to the stair vertex with the larger labeled line number.

Pattern IV. The stair vertex with the larger labeled line number $i+1$, shown as the right subfigure of Figure 5 remains contact with tracks, thus applying a force to the driving wheels. While the stair vertex with the smaller labeled line number $i$, shown as the right subfigure of Figure 5 remains no contact with tracks. The criterion for determining Pattern IV can be described as:

$$
\begin{gathered}
i \sqrt{h^{2}+b^{2}}+\frac{\left(r_{E}-r_{A}\right) \sqrt{h^{2}+b^{2}}}{h} \leq l, \\
\eta \leq r_{E} \quad \text { or } \quad \zeta \geq \frac{\pi}{2} .
\end{gathered}
$$




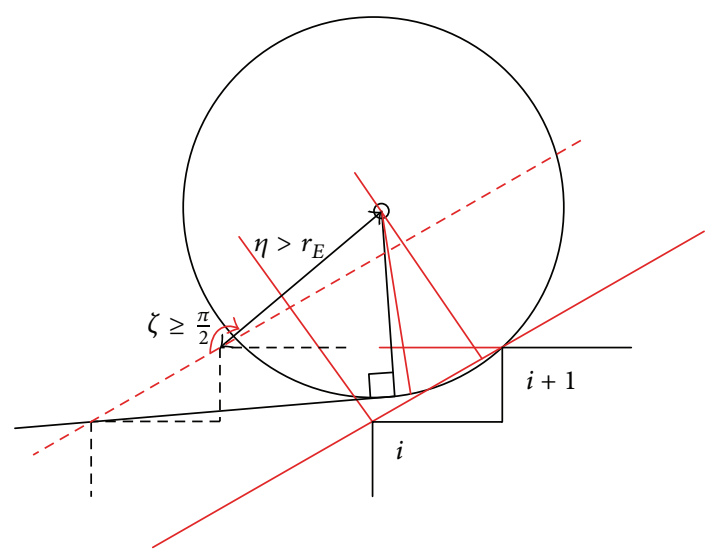

Figure 6: Pattern IV which meets the situation of $\eta>r_{E}$.

If the expression $\zeta<\pi / 2$ does not hold for the criterion (8) for determining Pattern III, as shown in Figure 6, Pattern IV might be mistaken for Pattern III.

Experimental results show that the stair-climbing process of WT wheelchair robot on each stair can be divided into a sequence of determinations for Pattern I, Pattern II, Pattern III, and Pattern IV, thus forming a simple cycle, as shown in Figure 7.

3.3. Analysis of the Pattern for the First Stair. The criterion for the robot climbing in the first stair is $\left(r_{E}-r_{A}\right) \sqrt{h^{2}+b^{2}} / h<l<$ $\sqrt{h^{2}+b^{2}}+r_{E}(b / h)-r_{A} \sqrt{h^{2}+b^{2}} / h$, with the wheelchair being in Pattern IV, as shown in Figure 8. When $l \geq \sqrt{h^{2}+b^{2}}+$ $r_{E}(b / h)-r_{A} \sqrt{h^{2}+b^{2}} / h$, the flow in Figure 7 can be used to determine the pattern.

\section{Active Tension Control by Using a Novel Control Law for Holonomic or Nonholonomic Systems}

The simplified dynamic model of WT wheelchair robot in each pattern can be described as follows.

Dynamic equation:

$$
\begin{gathered}
\frac{\mathrm{d}}{\mathrm{d} t}\left(\left[\begin{array}{c}
\frac{\partial \mathrm{Lag}}{\partial \dot{q}_{1}} \\
\frac{\partial \mathrm{Lag}}{\partial \dot{q}_{2}} \\
\frac{\partial \mathrm{Lag}}{\partial \dot{q}_{4}}
\end{array}\right]\right)-\left[\begin{array}{c}
\frac{\partial \mathrm{Lag}}{\partial q_{1}} \\
\frac{\partial \mathrm{Lag}}{\partial q_{2}} \\
\frac{\partial \mathrm{Lag}}{\partial q_{4}}
\end{array}\right] \\
-\left[\begin{array}{c}
\frac{\partial f\left(q_{1}, q_{2}\right)}{\partial q_{1}} \\
\frac{\partial f\left(q_{1}, q_{2}\right)}{\partial q_{2}} \\
0
\end{array}\right] 2 \lambda=\left[\begin{array}{c}
Q_{1} \\
2 Q_{2} \\
2 Q_{4}
\end{array}\right],
\end{gathered}
$$

where the Lagrangian is $\operatorname{Lag}=\operatorname{Lag}\left(q_{1}, \dot{q}_{1}, q_{2}, \dot{q}_{2}, q_{4}, \dot{q}_{4}\right)=$ $2 T_{E}+T_{B E}+T_{A B}+2 T_{C D}-2 V_{E}-V_{B E}-V_{A B}-2 V_{C D}$.
And the constraint equation is the same as (1):

$$
f\left(q_{1}, q_{2}\right)=0 \text {. }
$$

There are three generalized coordinates and a holonomic constraint in the system of WT wheelchair robot, so the wheelchair robot possesses two independent generalized coordinates and the number of degrees of freedom of the system is two. The Lagrange multiplier $\lambda$ corresponds to the constraint force of the holonomic constraint, namely, tension force of track. There are three power sources: $Q_{1}$ is the torque provided by the motor of the back flipper, $Q_{2}$ is the torque provided by the motor of front flippers, and $Q_{4}$ is the torque provided by the walking motors of driving wheels, which are used to control the wheelchair's generalized coordinates and constraint force. From above, one can know that the number of the system's power sources is equal to the sum of the number of the degrees of freedom and the number of the independent constraints, which is different from the control available for underactuated robots.

The following introduced theorem deals with such active tension control law for holonomic or nonholonomic robots.

Theorem 1. Assume that there are $n$ generalized coordinates and $g$ independent constraints in holonomic system. Dynamic equation based on the general form of the Hamilton principle of the system is as follows: $\int_{t_{0}}^{t_{1}}(\mathbf{D} \ddot{\mathbf{q}}+\mathbf{C} \dot{\mathbf{q}}+\mathbf{G}-\mathbf{Q})^{T} \delta \mathbf{q} \mathrm{d} t=$ $\mathbf{0}$, where the matrix $\mathbf{D} \in \mathbb{R}^{n \times n}$ is known as the inertial terms, the matrix $\mathbf{C} \in \mathbb{R}^{n \times n}$ is known as the centripetal and Coriolis forces, the matrix $\mathbf{G} \in \mathbb{R}^{n \times 1}$ is known as the gravity terms, and the matrix $\mathbf{Q} \in \mathbb{R}^{n \times 1}$ is known as the generalized forces corresponding to the torques driven by the motors of robot's joints. Let constraint equation be $\mathbf{f}(\mathbf{q}, t)=$ $\mathbf{0}$, where $\mathbf{f}(\bullet) \in \mathbb{R}^{g \times 1}$ is a vector function. So, the number of the degrees of freedom of the system is $k=n-g$, and thus there are $k$ independent generalized coordinates, which can be defined as $\mathbf{q}_{k}=\left[q_{1}, q_{2}, \ldots, q_{k}\right]^{T} \in \mathbb{R}^{k \times 1}$, while $\mathbf{q}_{g}=\left[q_{k+1}, q_{k+2}, \ldots, q_{n}\right]^{T} \in \mathbb{R}^{g \times 1}$ with $\mathbf{D}_{k} \in \mathbb{R}^{n \times k}$ are $k$ columns of the matrix $\mathbf{D}$ corresponding to $\ddot{\mathbf{q}}_{k}$, and $\mathbf{D}_{g} \in$ $\mathbb{R}^{n \times g}$ are $g$ columns of the matrix $\mathbf{D}$ corresponding to $\ddot{\mathbf{q}}_{g}$. If $\left[\mathbf{D}_{k}-\mathbf{D}_{g}\left(\partial \mathbf{f} / \partial \mathbf{q}_{g}^{T}\right)^{-1} \partial \mathbf{f} / \partial \mathbf{q}_{k}^{T} \mid-\partial \mathbf{f}^{T} / \partial \mathbf{q}\right] \in \mathbb{R}^{n \times n}$ is an invertible array, all of the generalized coordinates including independent generalized coordinates and dependent ones of a holonomic system can be tracked, and also constraint forces of holonomic constraints can be controlled, with a control law givenin the following:

$$
\begin{aligned}
\mathbf{Q}= & -\mathbf{D}_{g}\left(\frac{\partial \mathbf{f}}{\partial \mathbf{q}_{g}^{T}}\right)^{-1}\left(\mathbf{c f} \dot{\mathbf{q}}+\frac{\partial^{2} \mathbf{f}}{\partial t \partial \mathbf{q}^{T}} \dot{\mathbf{q}}+\frac{\partial^{2} \mathbf{f}}{\partial t^{2}}\right)+\mathbf{C} \dot{\mathbf{q}}+\mathbf{G} \\
& +\left[\mathbf{D}_{k}-\mathbf{D}_{g}\left(\frac{\partial \mathbf{f}}{\partial \mathbf{q}_{g}^{T}}\right)^{-1} \frac{\partial \mathbf{f}}{\partial \mathbf{q}_{k}^{T}} \mid-\frac{\partial \mathbf{f}^{T}}{\partial \mathbf{q}}\right] \\
& \times\left[\begin{array}{c}
\left.\ddot{\mathbf{q}}_{k d}+\mathbf{k}_{D} \dot{\mathbf{e}}_{k}+\mathbf{k}_{P} \mathbf{e}_{k}\right] . \\
\lambda_{d}
\end{array}\right]
\end{aligned}
$$




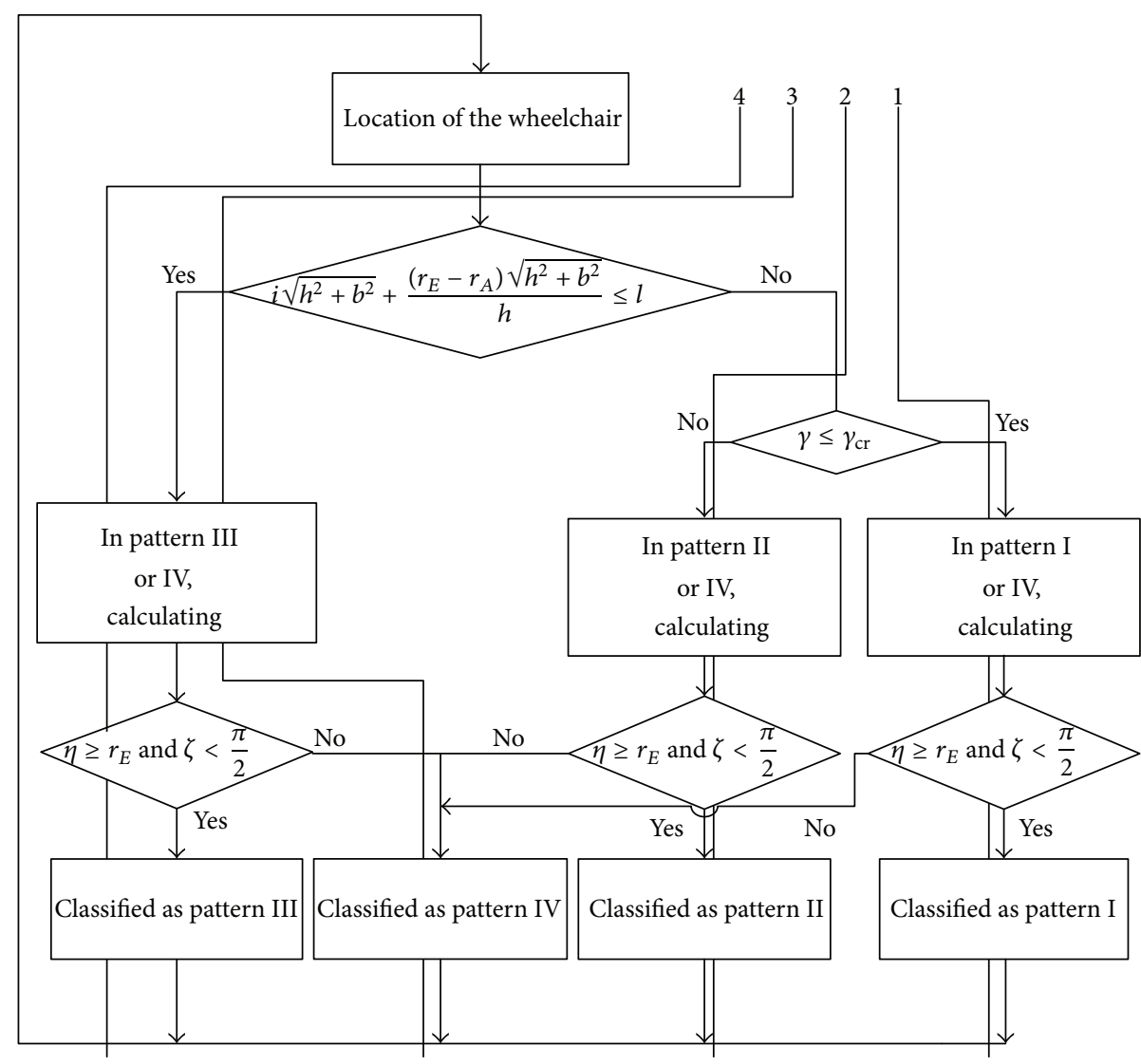

FIGURE 7: Flow chart of the determination of patterns.

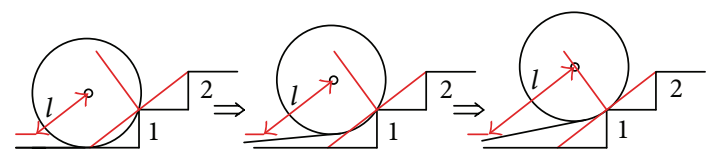

Figure 8: Climbing the first stair.

Proof. According to $g$, independent holonomic constraint equations, one can obtain the following:

$$
\mathbf{f}(\mathbf{q}, t)=\mathbf{0},
$$

that is $\mathbf{f}\left(\mathbf{q}_{k}, \mathbf{q}_{g}, t\right)=\mathbf{0}$.

According to implicit function theorem one gets the following:

$$
\mathbf{q}_{g}=\mathbf{q}_{g}\left(\mathbf{q}_{k}, t\right)
$$

Differentiating both sides of (13) with respect to the time $t$ yields

$$
\frac{\partial \mathbf{f}}{\partial \mathbf{q}^{T}} \dot{\mathbf{q}}+\frac{\partial \mathbf{f}}{\partial t}=\mathbf{0}
$$

that is,

$$
\left[\frac{\partial \mathbf{f}}{\partial \mathbf{q}_{k}^{T}} \mid \frac{\partial \mathbf{f}}{\partial \mathbf{q}_{g}^{T}}\right]\left[\begin{array}{c}
\dot{\mathbf{q}}_{k} \\
\dot{\mathbf{q}}_{g}
\end{array}\right]+\frac{\partial \mathbf{f}}{\partial t}=\mathbf{0} .
$$

Expanding it, one can obtain the following:

$$
\frac{\partial \mathbf{f}}{\partial \mathbf{q}_{k}^{T}} \dot{\mathbf{q}}_{k}+\frac{\partial \mathbf{f}}{\partial \mathbf{q}_{g}^{T}} \dot{\mathbf{q}}_{g}+\frac{\partial \mathbf{f}}{\partial t}=\mathbf{0} .
$$

Because (13) is independent, $\partial \mathbf{f} / \partial \mathbf{q}_{g}^{T} \in \mathbb{R}^{g \times g}$ is invertible and according to (17) one obtains the following:

$$
\dot{\mathbf{q}}_{g}=-\left(\frac{\partial \mathbf{f}}{\partial \mathbf{q}_{g}^{T}}\right)^{-1}\left(\frac{\partial \mathbf{f}}{\partial \mathbf{q}_{k}^{T}} \dot{\mathbf{q}}_{k}+\frac{\partial \mathbf{f}}{\partial t}\right) .
$$

Differentiating both sides of (15) with respect to the time $t$, one can obtain the following:

$$
\left[\begin{array}{c}
\dot{\mathbf{q}}^{T} \frac{\partial^{2} f_{1}}{\partial \mathbf{q}^{T} \partial \mathbf{q}} \\
\dot{\mathbf{q}}^{T} \frac{\partial^{2} f_{2}}{\partial \mathbf{q}^{T} \partial \mathbf{q}} \\
\vdots \\
\dot{\mathbf{q}}^{T} \frac{\partial^{2} f_{g}}{\partial \mathbf{q}^{T} \partial \mathbf{q}}
\end{array}\right] \dot{\mathbf{q}}+\frac{\partial \mathbf{f}}{\partial \mathbf{q}^{T}} \ddot{\mathbf{q}}+\frac{\partial^{2} \mathbf{f}}{\partial t \partial \mathbf{q}^{T}} \dot{\mathbf{q}}+\frac{\partial^{2} \mathbf{f}}{\partial t^{2}}=\mathbf{0},
$$


where one can define the following:

$$
\mathbf{c f}=\left[\begin{array}{c}
\dot{\mathbf{q}}^{T} \frac{\partial^{2} f_{1}}{\partial \mathbf{q}^{T} \partial \mathbf{q}} \\
\dot{\mathbf{q}}^{T} \frac{\partial^{2} f_{2}}{\partial \mathbf{q}^{T} \partial \mathbf{q}} \\
\vdots \\
\dot{\mathbf{q}}^{T} \frac{\partial^{2} f_{g}}{\partial \mathbf{q}^{T} \partial \mathbf{q}}
\end{array}\right] \in \mathbb{R}^{g \times n},
$$

and thus substituting it into (19), one can obtain the following: $\left(\partial \mathbf{f} / \partial \mathbf{q}^{T}\right) \ddot{\mathbf{q}}+\mathbf{c f} \dot{\mathbf{q}}+\left(\partial^{2} \mathbf{f} / \partial t \partial \mathbf{q}^{T}\right) \dot{\mathbf{q}}+\partial^{2} \mathbf{f} / \partial t^{2}=\mathbf{0}$,

$$
\begin{gathered}
{\left[\frac{\partial \mathbf{f}}{\partial \mathbf{q}_{k}^{T}} \mid \frac{\partial \mathbf{f}}{\partial \mathbf{q}_{g}^{T}}\right]\left[\begin{array}{c}
\ddot{\mathbf{q}}_{k} \\
\ddot{\mathbf{q}}_{g}
\end{array}\right]+\mathbf{c f} \dot{\mathbf{q}}+\frac{\partial^{2} \mathbf{f}}{\partial t \partial \mathbf{q}^{T}} \dot{\mathbf{q}}+\frac{\partial^{2} \mathbf{f}}{\partial t^{2}}=\mathbf{0},} \\
\frac{\partial \mathbf{f}}{\partial \mathbf{q}_{k}^{T}} \ddot{\mathbf{q}}_{k}+\frac{\partial \mathbf{f}}{\partial \mathbf{q}_{g}^{T}} \ddot{\mathbf{q}}_{g}+\mathbf{c f} \dot{\mathbf{q}}+\frac{\partial^{2} \mathbf{f}}{\partial t \partial \mathbf{q}^{T}} \dot{\mathbf{q}}+\frac{\partial^{2} \mathbf{f}}{\partial t^{2}}=\mathbf{0},
\end{gathered}
$$

and thus

$$
\ddot{\mathbf{q}}_{g}=-\left(\frac{\partial \mathbf{f}}{\partial \mathbf{q}_{g}^{T}}\right)^{-1}\left(\frac{\partial \mathbf{f}}{\partial \mathbf{q}_{k}^{T}} \ddot{\mathbf{q}}_{k}+\mathbf{c f} \dot{\mathbf{q}}+\frac{\partial^{2} \mathbf{f}}{\partial t \partial \mathbf{q}^{T}} \dot{\mathbf{q}}+\frac{\partial^{2} \mathbf{f}}{\partial t^{2}}\right)
$$

According to (15), the variation of generalized coordinates of constraint equations can be described as $\left(\partial \mathbf{f} / \partial \mathbf{q}^{T}\right) \delta \mathbf{q}=\mathbf{0}$. Multiplying it by the undetermined coefficient $\lambda \in \mathbb{R}^{g \times 1}$ and adding it to the dynamic equations, one can obtain the following:

$$
\int_{t_{0}}^{t_{1}}\left(\mathbf{D} \ddot{\mathbf{q}}+\mathbf{C} \dot{\mathbf{q}}+\mathbf{G}-\frac{\partial \mathbf{f}^{T}}{\partial \mathbf{q}} \boldsymbol{\lambda}-\mathbf{Q}\right)^{T} \delta \mathbf{q} \mathrm{d} t=\mathbf{0},
$$

where there are $k=n-g$ independent coordinates of the system, and also one can adjust $g$, other undetermined multipliers so that the coefficients in front of the dependent coordinates of the system remain 0 , with a result obtained as $\mathbf{D} \ddot{\mathbf{q}}+\mathbf{C} \dot{\mathbf{q}}+\mathbf{G}-\left(\partial \mathbf{f}^{T} / \partial \mathbf{q}\right) \boldsymbol{\lambda}-\mathbf{Q}=\mathbf{0}$. Expanding it gives the following:

$$
\left[\mathbf{D}_{k} \mid \mathbf{D}_{g}\right]\left[\begin{array}{c}
\ddot{\mathbf{q}}_{k} \\
\ddot{\mathbf{q}}_{g}
\end{array}\right]+\mathbf{C} \dot{\mathbf{q}}+\mathbf{G}-\frac{\partial \mathbf{f}^{T}}{\partial \mathbf{q}} \boldsymbol{\lambda}=\mathbf{Q},
$$

and thus

$$
\mathbf{D}_{k} \ddot{\mathbf{q}}_{k}+\mathbf{D}_{g} \ddot{\mathbf{q}}_{g}+\mathbf{C} \dot{\mathbf{q}}+\mathbf{G}-\frac{\partial \mathbf{f}^{T}}{\partial \mathbf{q}} \boldsymbol{\lambda}=\mathbf{Q}
$$

Substituting for $\ddot{\mathbf{q}}_{g}$ from (22) into (25) yields the following:

$$
\begin{aligned}
\mathbf{D}_{k} \ddot{\mathbf{q}}_{k} & -\mathbf{D}_{g}\left(\frac{\partial \mathbf{f}}{\partial \mathbf{q}_{g}^{T}}\right)^{-1}\left(\frac{\partial \mathbf{f}}{\partial \mathbf{q}_{k}^{T}} \ddot{\mathbf{q}}_{k}+\mathbf{c f} \dot{\mathbf{q}}+\frac{\partial^{2} \mathbf{f}}{\partial t \partial \mathbf{q}^{T}} \dot{\mathbf{q}}+\frac{\partial^{2} \mathbf{f}}{\partial t^{2}}\right) \\
& +\mathbf{C} \dot{\mathbf{q}}+\mathbf{G}-\frac{\partial \mathbf{f}^{T}}{\partial \mathbf{q}} \lambda=\mathbf{Q} .
\end{aligned}
$$

Substituting for $\dot{\mathbf{q}}_{g}$ from (18) into (26) and then substituting for $\mathbf{q}_{g}$ from (14) into (26), one can obtain the following:

$$
\begin{aligned}
{\left[\mathbf{D}_{k}\right.} & \left.-\mathbf{D}_{g}\left(\frac{\partial \mathbf{f}}{\partial \mathbf{q}_{g}^{T}}\right)^{-1} \frac{\partial \mathbf{f}}{\partial \mathbf{q}_{k}^{T}}\right] \ddot{\mathbf{q}}_{k}-\mathbf{D}_{g}\left(\frac{\partial \mathbf{f}}{\partial \mathbf{q}_{g}^{T}}\right)^{-1} \\
& \times\left(\mathbf{c f} \dot{\mathbf{q}}+\frac{\partial^{2} \mathbf{f}}{\partial t \partial \mathbf{q}^{T}} \dot{\mathbf{q}}+\frac{\partial^{2} \mathbf{f}}{\partial t^{2}}\right)+\mathbf{C} \dot{\mathbf{q}}+\mathbf{G}-\frac{\partial \mathbf{f}^{T}}{\partial \mathbf{q}} \boldsymbol{\lambda}=\mathbf{Q} .
\end{aligned}
$$

A dynamic equation containing only the independent generalized coordinates $\mathbf{q}_{k}$ and the Lagrange multipliers $\lambda$ can be obtained as follows:

$$
\begin{gathered}
{\left[\mathbf{D}_{k}-\mathbf{D}_{g}\left(\frac{\partial \mathbf{f}}{\partial \mathbf{q}_{g}^{T}}\right)^{-1} \frac{\partial \mathbf{f}}{\partial \mathbf{q}_{k}^{T}} \mid-\frac{\partial \mathbf{f}^{T}}{\partial \mathbf{q}}\right]\left[\begin{array}{c}
\ddot{\mathbf{q}}_{k} \\
\lambda
\end{array}\right]} \\
\quad-\mathbf{D}_{g}\left(\frac{\partial \mathbf{f}}{\partial \mathbf{q}_{g}^{T}}\right)^{-1}\left(\mathbf{c f} \dot{\mathbf{q}}+\frac{\partial^{2} \mathbf{f}}{\partial t \partial \mathbf{q}^{T}} \dot{\mathbf{q}}+\frac{\partial^{2} \mathbf{f}}{\partial t^{2}}\right) \\
+\mathbf{C} \dot{\mathbf{q}}+\mathbf{G}=\mathbf{Q} .
\end{gathered}
$$

A control law can be designed as (12), where $\mathbf{q}_{k d}, \dot{\mathbf{q}}_{k d}$, and $\ddot{\mathbf{q}}_{k d}$ are known as the expected values of independent generalized coordinates of $\mathbf{q}_{k}, \dot{\mathbf{q}}_{k}$, and $\ddot{\mathbf{q}}_{k}$, thus difference functions of independent generalized coordinates being defined as $\mathbf{e}_{k}=$ $\mathbf{q}_{k d}-\mathbf{q}_{k}, \dot{\mathbf{e}}_{k}=\dot{\mathbf{q}}_{k d}-\dot{\mathbf{q}}_{k}$, and $\ddot{\mathbf{e}}_{k}=\ddot{\mathbf{q}}_{k d}-\ddot{\mathbf{q}}_{k}$ with $\mathbf{k}_{D}$ and $\mathbf{k}_{P}$ being positive definite matrices which can be selected.

Substituting for such control law or the torque vector $\mathbf{Q}$ from (12) into the dynamic equation (28) yields the following:

$$
\begin{aligned}
& {\left[\mathbf{D}_{k}-\mathbf{D}_{g}\left(\frac{\partial \mathbf{f}}{\partial \mathbf{q}_{g}^{T}}\right)^{-1} \frac{\partial \mathbf{f}}{\partial \mathbf{q}_{k}^{T}} \mid-\frac{\partial \mathbf{f}^{T}}{\partial \mathbf{q}}\right]} \\
& \times\left[\begin{array}{c}
\ddot{\mathbf{q}}_{k}-\ddot{\mathbf{q}}_{k d}-\mathbf{k}_{D} \dot{\mathbf{e}}_{k}-\mathbf{k}_{P} \mathbf{e}_{k} \\
\lambda-\lambda_{d}
\end{array}\right]=\mathbf{0} .
\end{aligned}
$$

According to the assumption of the theorem, if the matrix

$$
\left[\mathbf{D}_{k}-\mathbf{D}_{g}\left(\frac{\partial \mathbf{f}}{\partial \mathbf{q}_{g}^{T}}\right)^{-1} \frac{\partial \mathbf{f}}{\partial \mathbf{q}_{k}^{T}} \mid-\frac{\partial \mathbf{f}^{T}}{\partial \mathbf{q}}\right]
$$

is invertible, (29) has only a zero solution as follows:

$$
\begin{gathered}
\ddot{\mathbf{e}}_{k}+\mathbf{k}_{D} \dot{\mathbf{e}}_{k}+\mathbf{k}_{P} \mathbf{e}_{k}=\mathbf{0}, \\
\lambda=\lambda_{d} .
\end{gathered}
$$




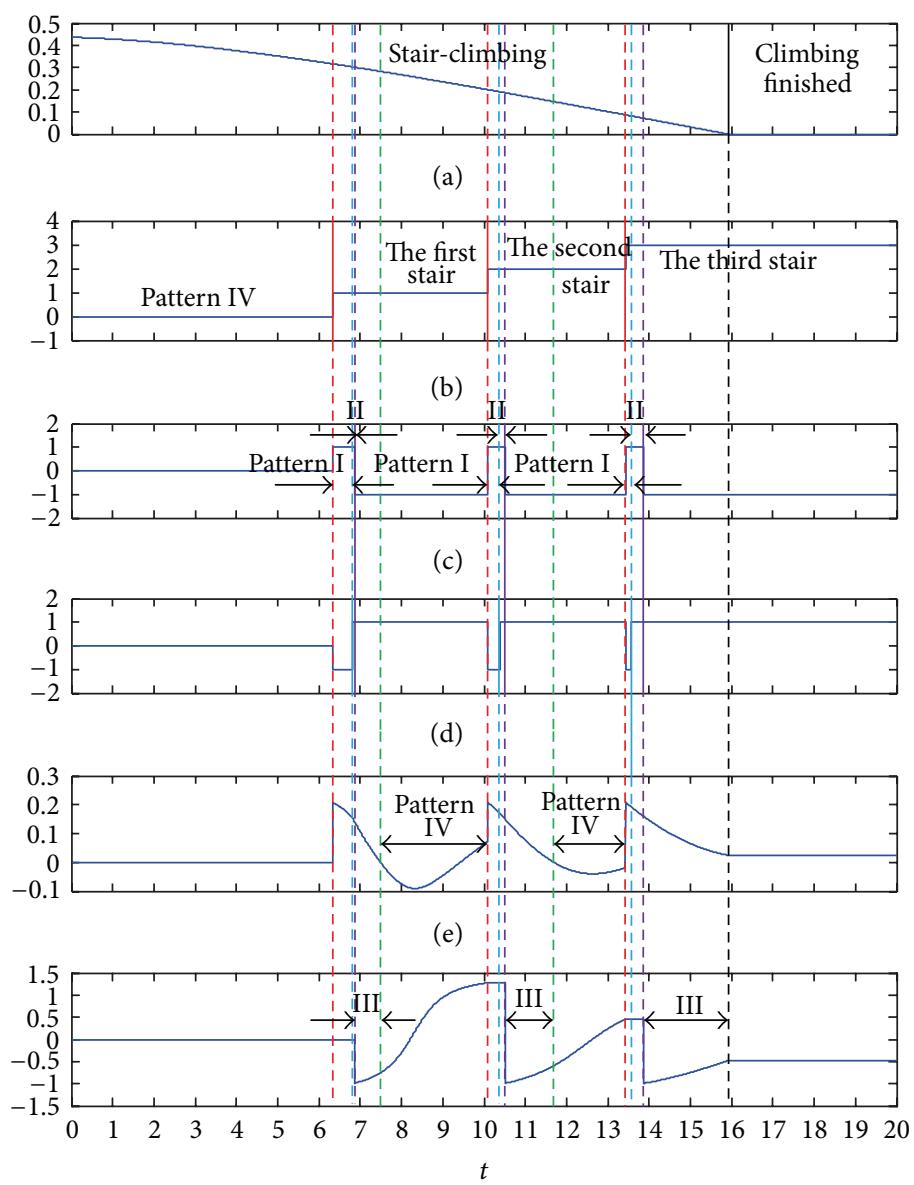

(f)

FIGURE 9: Schematics diagram of simulation results by using stateflow.

According to $\lambda=\lambda_{d}$, the control law can be used to control constraint forces of holonomic constraints of the system. And also the Lyapunov function can be proposed as $V=(1 / 2) \dot{\mathbf{e}}_{k}^{T} \dot{\mathbf{e}}_{k}+(1 / 2) \mathbf{e}_{k}^{T} \mathbf{k}_{P} \mathbf{e}_{k}$. Because $\mathbf{k}_{P}$ is positive definite, one can obtain $V>0$, and differentiating a both sides of the Lyapunov function with respect to the time $t$ one can obtain the following:

$$
\dot{V}=\dot{\mathbf{e}}_{k}^{T} \ddot{\mathbf{e}}_{k}+\dot{\mathbf{e}}_{k}^{T} \mathbf{k}_{P} \mathbf{e}_{k}=\dot{\mathbf{e}}_{k}^{T}\left(\ddot{\mathbf{e}}_{k}+\mathbf{k}_{P} \mathbf{e}_{k}\right) .
$$

Substituting $\ddot{\mathbf{e}}_{k}+\mathbf{k}_{P} \mathbf{e}_{k}=\mathbf{k}_{D} \dot{\mathbf{e}}_{k}$, where $\mathbf{k}_{D}$ is a positive definite, from (31) into the derivative of the Lyapunov function (32), one can obtain $\dot{V}=-\dot{\mathbf{e}}_{k}^{T} \mathbf{k}_{D} \dot{\mathbf{e}}_{k}<\mathbf{0}$, which means the system is stable and that $\lim _{t \rightarrow \infty} \mathbf{e}_{k}=\mathbf{0}$.

By using the control law (12), one can make the controlled independent generalized coordinates $\mathbf{q}_{k}$ track the expected reference input $\mathbf{q}_{k d}$, and the relative curves of dependent generalized coordinates $\mathbf{q}_{g}$ can be obtained by (14), (18), and (22).

Just making an appropriate change of constraint equations, one can design the control law for the nonholonomic system by using the above theorem.
Applying the above theorem to WT wheelchair robot, with (10) and (11), one can obtain the control law for the system as follows:

$$
\begin{aligned}
{\left[\begin{array}{c}
Q_{1} \\
2 Q_{2} \\
2 Q_{4}
\end{array}\right]=} & -\mathbf{D}_{2}\left(\frac{\partial f}{\partial q_{2}}\right)^{-1} \mathbf{c f} \dot{\mathbf{q}}+\mathbf{C} \dot{\mathbf{q}}+\mathbf{G} \\
& +\left[\mathbf{D}_{1}-\mathbf{D}_{2}\left(\frac{\partial f}{\partial q_{2}}\right)^{-1} \frac{\partial f}{\partial q_{1}}\left|-\frac{\partial f}{\partial \mathbf{q}}\right| \mathbf{D}_{4}\right] \\
& \times\left[\begin{array}{c}
\ddot{q}_{1 d}+k_{D 1}\left(\dot{q}_{1 d}-\dot{q}_{1}\right)+k_{P 1}\left(q_{1 d}-q_{1}\right) \\
2 \lambda_{d} \\
\ddot{q}_{4 d}+k_{D 4}\left(\dot{q}_{4 d}-\dot{q}_{4}\right)+k_{P 4}\left(q_{4 d}-q_{4}\right)
\end{array}\right],
\end{aligned}
$$

where $\mathbf{D}_{1}, \mathbf{D}_{2}$, and $\mathbf{D}_{4}$ denote the first column, the second column, and the third column of the inertia terms $\mathbf{D}$.

Substituting the control law (33) with the dynamic equation (10) of the wheelchair, one can obtain the differential 


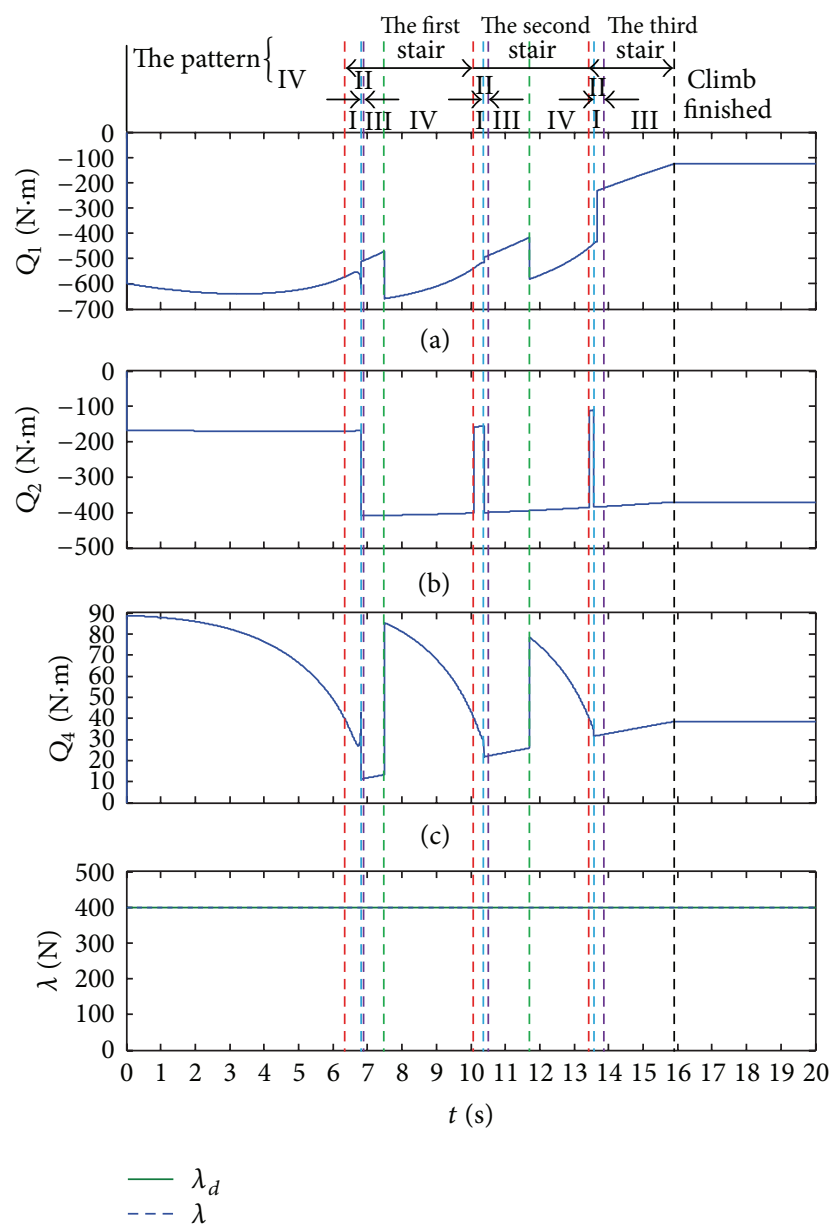

(d)

FIGURE 10: Torque of each joint provided by motor and the Lagrange multiplier.

equations corresponding to the independent generalized coordinates $q_{1}$ and $q_{4}$ and the constraint force $\lambda$ as follows:

$$
\begin{gathered}
\ddot{q}_{1}=\ddot{q}_{1 d}+k_{D 1}\left(\dot{q}_{1 d}-\dot{q}_{1}\right)+k_{P 1}\left(q_{1 d}-q_{1}\right), \\
2 \lambda=2 \lambda_{d}, \\
\ddot{q}_{4}=\ddot{q}_{4 d}+k_{D 4}\left(\dot{q}_{4 d}-\dot{q}_{4}\right)+k_{P 4}\left(q_{4 d}-q_{4}\right) .
\end{gathered}
$$

\section{Analysis of Simulation and Experiment}

Using the module stateflow [21] in MATLAB to simulate the entire stair-climbing process for WT wheelchair robot, one can obtain simulation curves about the determination of patterns, motion trajectories of the wheelchair, and the torques provided by each joint's motor and the tension force provided by the holonomic constraint of the track of the wheelchair.

5.1. Simulation Curves for the Determination of Patterns. An effective determination of pattern for WT wheelchair robot during stair-climbing process may need some parameter

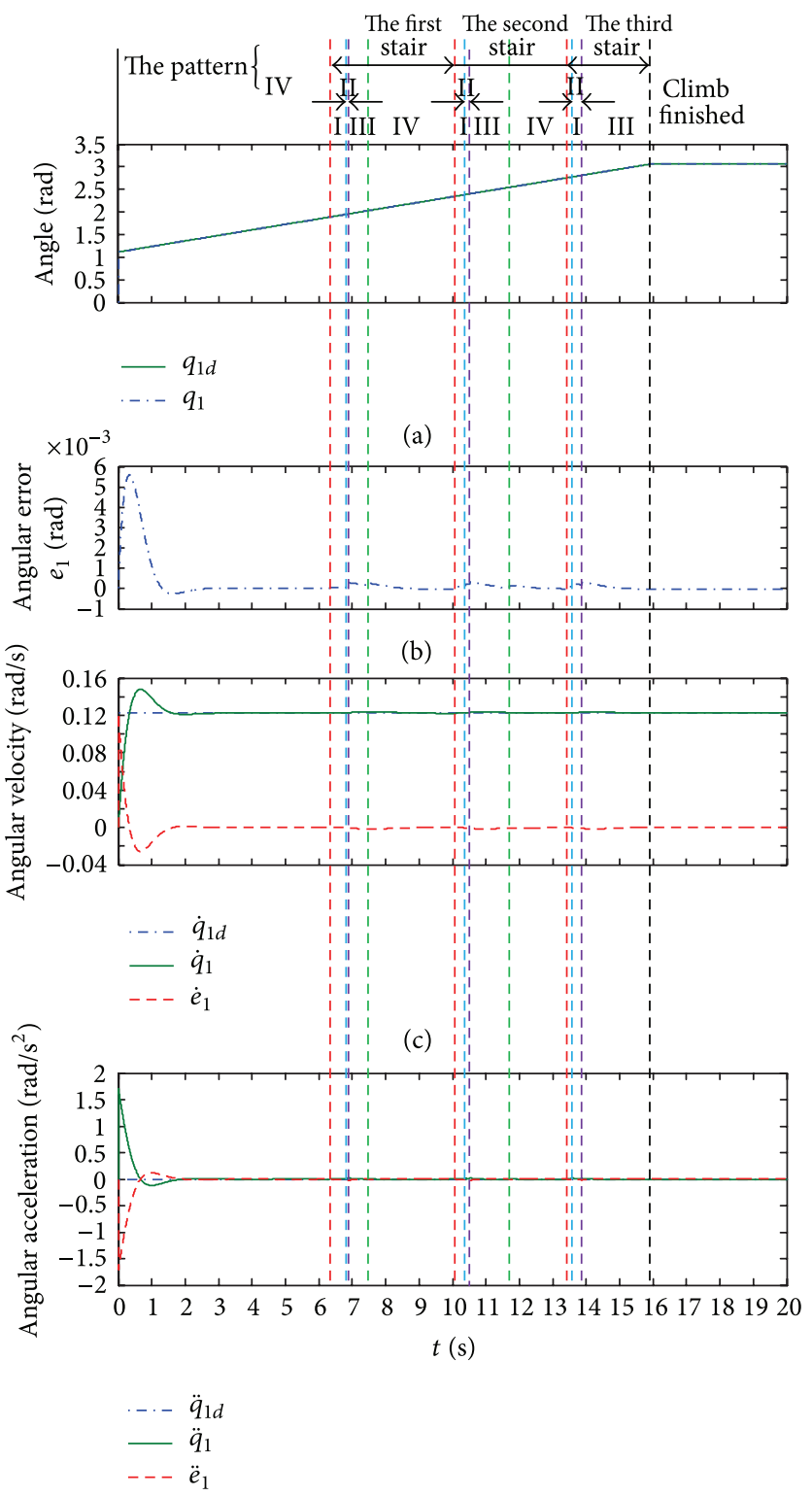

(d)

FIGURE 11: Curve of the independent generalized coordinate $q_{1}$ and that of its reference input $q_{1 d}$.

curves together. And according to the decision-making flow given in Figure 7, one can obtain analytical results of the entire stair-climbing process, as shown in Figure 9.

All abscissas of the curves in Figure 9 are the time $t$, second in unit. The ordinate of the curve in Figure 9(a) is $\gamma_{\mathrm{cr}}$, rad in unit, and the left of the black line at about $t=16$ (s) means "in the stair-climbing process" while the right of the black line means "the stair-climbing process finished." The ordinate of the curve in Figure 9(b) is $i$ without unit as is described as (4), with $i=1, i=2$, and $i=3$ denoting the section of stairs where wheelchair robot climbs. The ordinate of the curve in Figure 9(c) is the sign of $i \sqrt{h^{2}+b^{2}}+\left(\left(r_{E}-\right.\right.$ $\left.\left.r_{A}\right) \sqrt{h^{2}+b^{2}} / h\right)-l$ without unit as described in Section 2, with a value of 0 representing the climb of WT wheelchair robot 


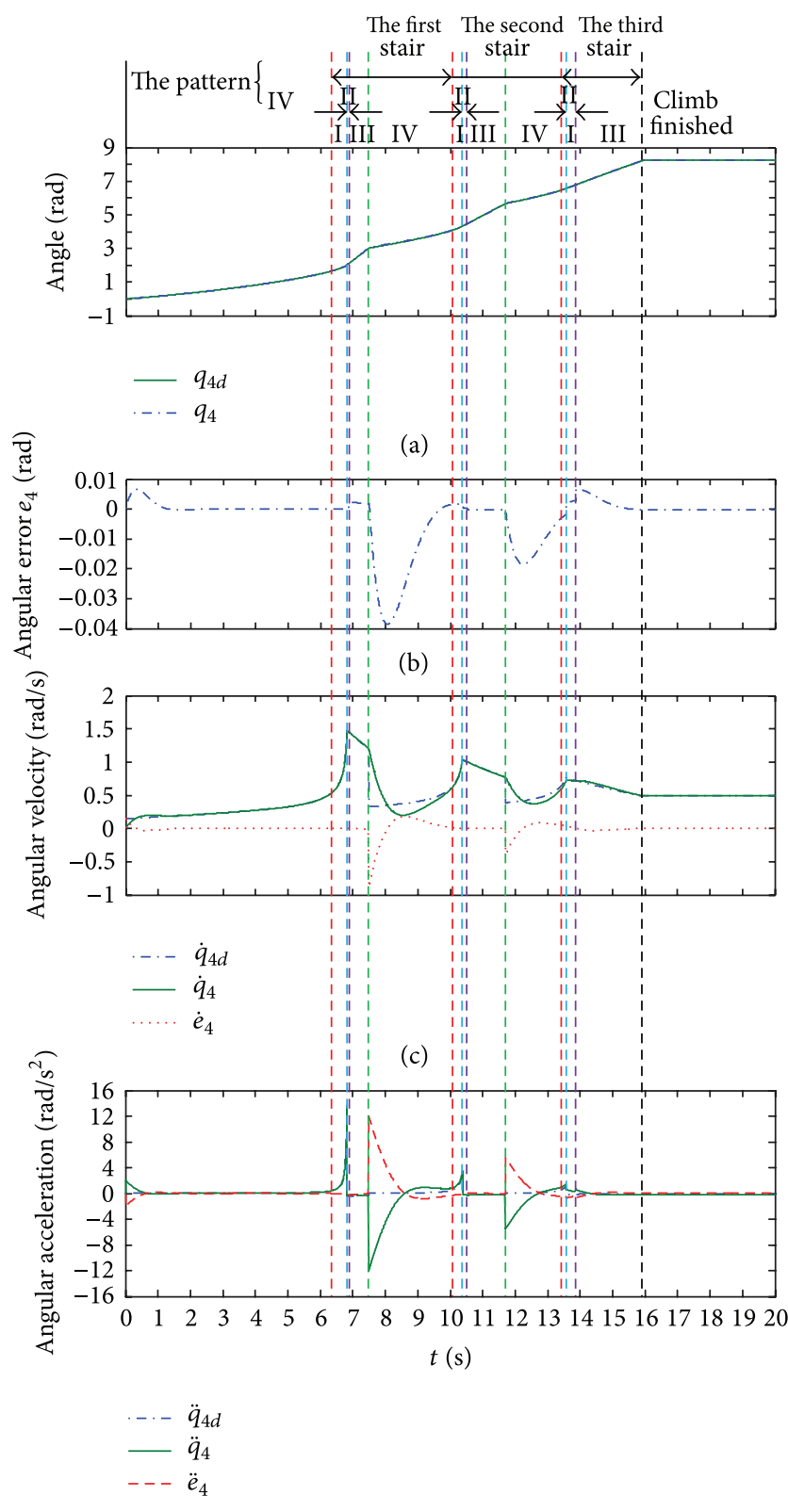

(d)

FIGURE 12: Curve of the independent generalized coordinate $q_{4}$ and that of its reference input $q_{4 d}$.

between the start and the first stair in Pattern IV, a value of 1 indicating the wheelchair robot is in Pattern I or II, or a value of -1 indicating the wheelchair robot is in Pattern III or IV. The ordinate of the curve in Figure 9(d) is the sign of $\gamma-\gamma_{\mathrm{cr}}$ without unit, with a combination with Figure 9(c) being made that a value of 1 indicates the range of Pattern I, and that a value of -1 indicates the range of Pattern II, as shown in Figures 9(c) and 9(d). The ordinate of the curve in Figure $9(\mathrm{e})$ is $\eta-r_{E}$, meter in unit, and the ordinate of the curve in Figure $9(\mathrm{f})$ is $\zeta-\pi / 2$, rad in unit. According to (8) and (9), the range of Pattern III and the range of Pattern IV can be determined, as shown in Figures 9(e) and 9(f).

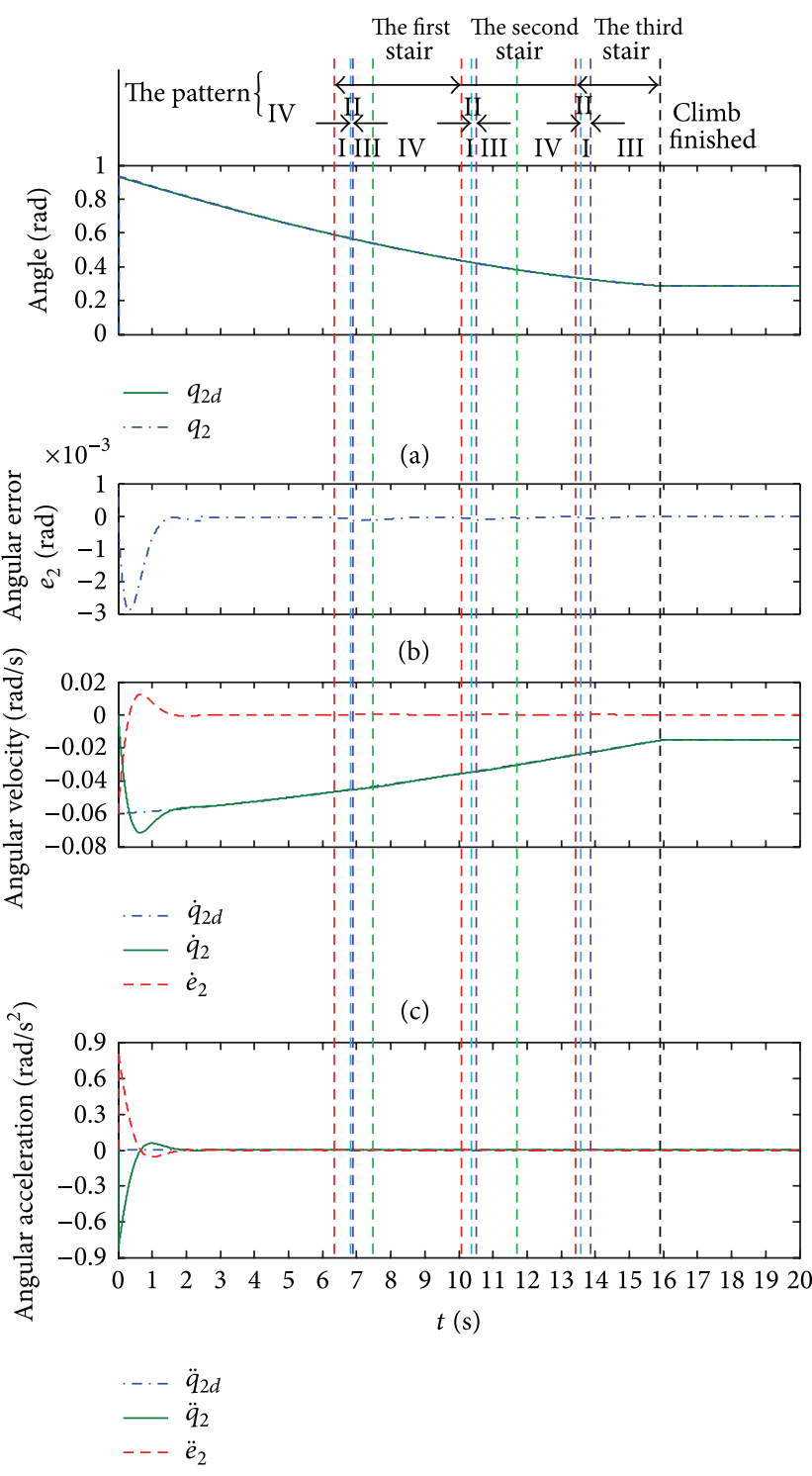

(d)

FIGURE 13: Curve of the independent generalized coordinate $q_{2}$ and that of its reference input $q_{2 d}$.

5.2. Simulation for Active Tension Control. In WT wheelchair robot system, the back flipper is controlled by a switch, which can be expressed by the generalized coordinates as $q_{1}=c 1 \cdot t+$ $q_{10}$, where $c 1=0.1222(\mathrm{rad} / \mathrm{s})$ is the constant angular velocity corresponding to the motor of the back flipper, $t$ is time, and $q_{10}=1.1132(\mathrm{rad})$ is the angle of the initial position. The seat plane of WT wheelchair robot needs to remain horizontal, namely, $q_{6}=q_{6}\left(q_{1}, q_{4}\right) \equiv 0$. Plus, the constraint equation (11) $f\left(q_{1}, q_{2}\right)=0$, one can obtain an equation set through as follows:

$$
\begin{gathered}
q_{1}=c 1 \cdot t+q_{10}, \\
q_{6}\left(q_{1}, q_{4}\right)=0, \\
f\left(q_{1}, q_{2}\right)=0,
\end{gathered}
$$




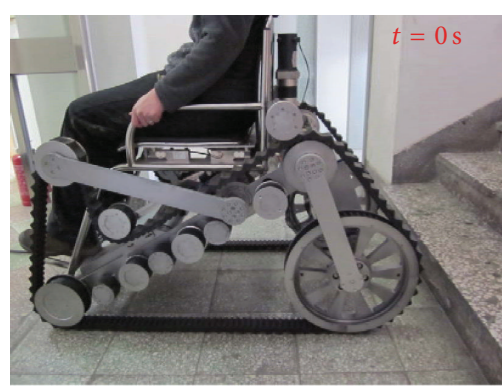

(a) $i=0$, start

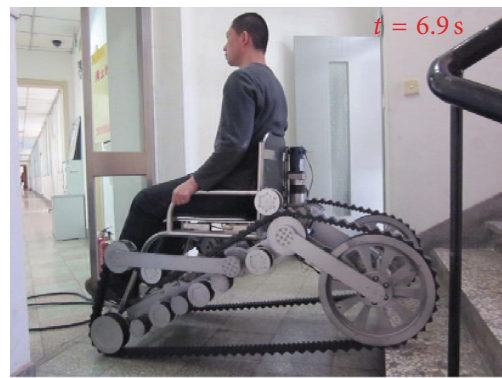

(d) $i=1$, pattern $=$ II

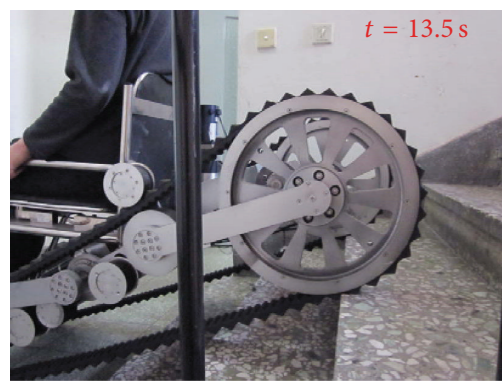

(g) $i=3$, pattern $=\mathrm{I}$

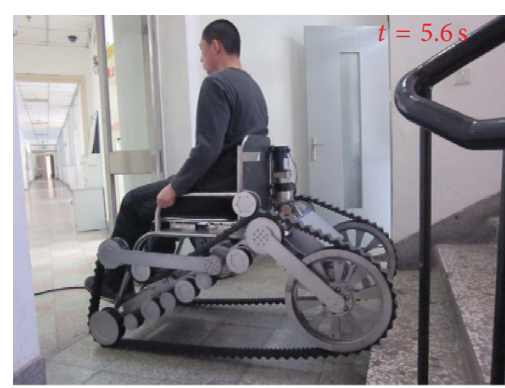

(b) $i=0$, pattern $=\mathrm{IV}$

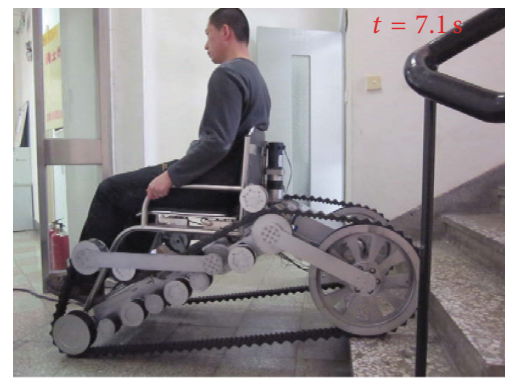

(e) $i=1$, pattern $=$ III

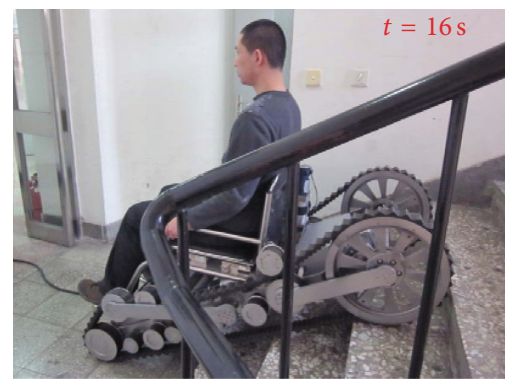

(h) $i=3$, pattern $=$ III, finished

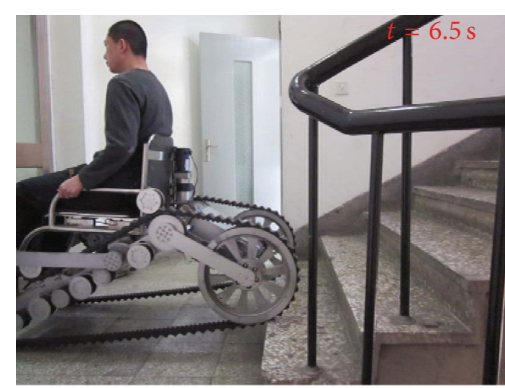

(c) $i=1$, pattern $=\mathrm{I}$

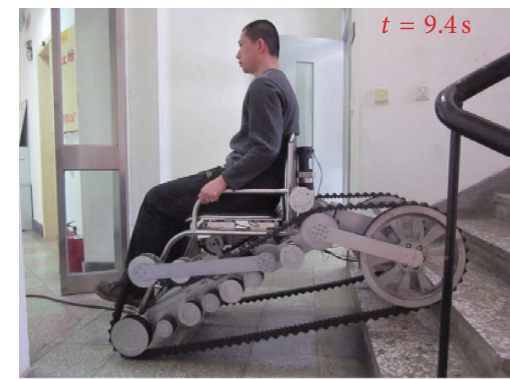

(f) $i=1$, pattern $=$ IV

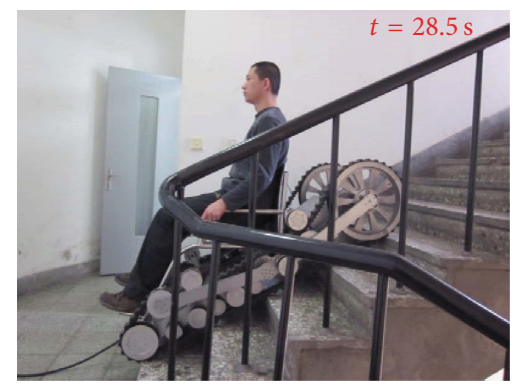

(i) Climb finished

FIGURE 14: Record of stair-climbing experiment for WT wheelchair robot.

from which one can obtain the reference inputs of the generalized coordinates $q_{1 d}, q_{2 d}, q_{4 d}$, and those of their derivatives.

Selecting positive definite matrices $\mathbf{k}_{P}=\left[\begin{array}{cc}k_{P 1} & 0 \\ 0 & k_{P_{4}}\end{array}\right]=$ $\left[\begin{array}{cc}100 & 0 \\ 0 & 100\end{array}\right]$ and $\mathbf{k}_{D}=\left[\begin{array}{cc}k_{D 1} & 0 \\ 0 & k_{D 4}\end{array}\right]=\left[\begin{array}{cc}14 & 0 \\ 0 & 14\end{array}\right]$, one can obtain the torque of each joint and the difference of the Lagrange multiplier $\lambda$ of the output constraint force from $\lambda_{d}$ of the expected input constraint force by using control law (33), as shown in Figure 10.

Figure 10(d) shows that according to such a control law that one can make the constraint force of the track of the wheelchair to follow the reference input tension, and that at the same time one can obtain the simulation curve of the motion trajectory of each expected reference input and the output curve acted by such a controller, as shown in Figures 11,12 , and 13 .

Because the time span of the stair-climbing process is much larger, the output curves where control law (12) is applied to the robot and the expected reference input curves are similar and are not distinguished easily, as shown in
Figures 11(a), 12(a), and 13(a). So, one can list their angular difference curves of joints, as shown in Figures 11(b), 12(b), and $13(b)$.

A comparison of the output curves of the wheelchair with the expected reference input curves of the wheelchair shows that by using such a control law one can make the output curves to follow the expected reference input curves immediately and then to remain stable.

5.3. Experiment of WT Wheelchair Robot in the Process of Stair-Climbing. The simulation curves about the torques can be used as a basis for the selection of motors. And Figure 14 demonstrates the entire experimental process of the WT wheelchair robot during the stair-climbing process. If the controller could make outputs $q_{1}, q_{2}$, and $q_{4}$ follow the reference inputs $q_{1 d}, q_{2 d}$, and $q_{4 d}$, then $q_{6}$ could follow the reference purpose $q_{6 d}=0$, which should mean that the plane angle of the seat corresponding to the horizontal plane follow 0 or the seat of the wheelchair maintains the horizontal angle, as shown in Figure 14 that the seat of the wheelchair 
varies around the horizontal surface, which can be regarded as an indirect reflection of the effectiveness of the designed controller.

\section{Conclusions}

Combined with the computed torque method, a novel control law applied to holonomic or nonholonomic systems is derived, with the active tension of the track controlled for WT wheelchair robot during the stair-climbing process simultaneously. The simulation of each of output and each expected reference input of the generalized coordinate shows the effectiveness of the proposed control law, the experimental results of the stair-climbing process for WT wheelchair robot verifying its obstacle-navigating performance, and the patterns analyzed above are embodied in the experiment.

\section{Acknowledgment}

This work was supported in part by the National Natural Science Foundation of China under Grant no. 60805048.

\section{References}

[1] T. Nishiyama, F. Takiuchi, K. Ando, and M. Arisawa, "The functional evaluation of future wheelchairs contributing to ecological aid in traveling," in Proceedings of the 4th International Symposium on Environmentally Conscious Design and Inverse Manufacturing (Eco Design '05), pp. 844-849, December 2005.

[2] Y. Sugahara, A. Ohta, K. Hashimoto et al., "Walking up and down stairs carrying a human by a biped locomotor with parallel mechanism," in Proceedings of the IEEE IRS/RSJ International Conference on Intelligent Robots and Systems (IROS '05), pp. 1489-1494, August 2005.

[3] J. J. Yuan, "Stability analyses of wheelchair robot based on "Humanin-the-Loop" control theory," in Proceedings of the IEEE International Conference on Robotics and Biomimetics, pp. 419424, Bankok, Thailand, February 2009.

[4] S. Hirose, E. F. Fukushima, R. Damoto, and H. Nakamoto, "Design of terrain adaptive versatile crawler vehicle HELIOSVI," in Proceedings of the IEEE/RSJ International Conference on Intelligent Robots and Systems, vol. 3, pp. 1540-1545, Maui, Hawaii, USA, October 2001.

[5] M. J. Lawn, T. Sakai, M. Kuroiwa, and T. Ishimatsu, "Development and practical application of a stairclimbing wheelchair in Nagasaki," Journal of HWRS-ERC, vol. 2, no. 2, pp. 33-39, 2001.

[6] I. Kolmanovsky and N. H. McClamroch, "Developments in nonholonomic control problems," IEEE Control Systems Magazine, vol. 15, no. 6, pp. 20-36, 1995.

[7] V. I. Arnold, V. V. Kozlov, and A. I. Neishtadt, "Mathematical aspects of classical and celestial mechanics," in Encyclopedia of Mathematical Sciences, V. I. Arnold, Ed., vol. 3 of Dynamical Systems, Springer, Berlin, Germany, 1988.

[8] N. H. McClamroch and D. Wang, "Feedback stabilization and tracking of constrained robots," IEEE Transactions on Automatic Control, vol. 33, no. 5, pp. 419-426, 1988.

[9] J. Yuan, "Adaptive control of a constrained robot-ensuring zero tracking and zero force errors," IEEE Transactions on Automatic Control, vol. 42, no. 12, pp. 1709-1714, 1997.
[10] C. S. Chiu, K. Y. Lian, and T. C. Wu, "Robust adaptive motion/force tracking control design for uncertain constrained robot manipulators," Automatica, vol. 40, no. 12, pp. 2111-2119, 2004.

[11] Y. C. Chang and B. S. Chen, "Intelligent robust tracking controls for holonomic and nonholonomic mechanical systems using only position measurements," IEEE Transactions on Fuzzy Systems, vol. 13, no. 4, pp. 491-507, 2005.

[12] Y. Zhao and C. C. Cheah, "Position and force control of robot manipulators using neural networks," in Proceedings of the IEEE Conference on Robotics, Automation and Mechatronics, vol. 1, pp. 300-305, Singapore, December 2004.

[13] W. Gueaieb, F. Karray, and S. Al-Sharhan, "A robust hybrid intelligent position/force control scheme for cooperative manipulators," IEEE/ASME Transactions on Mechatronics, vol. 12, no. 2, pp. 109-125, 2007.

[14] A. M. Bloch, M. Reyhanoglu, and N. H. McClamroch, "Control and stabilization of nonholonomic dynamic systems," IEEE Transactions on Automatic Control, vol. 37, no. 11, pp. 1746-1757, 1992.

[15] Z. Li, S. S. Ge, M. Adams, and W. S. Wijesoma, "Adaptive robust output-feedback motion/force control of electrically driven nonholonomic mobile manipulators," IEEE Transactions on Control Systems Technology, vol. 16, no. 6, pp. 1308-1315, 2008.

[16] C. Y. Su and Y. Stepanenko, "Robust motion/force control of mechanical systems with classical nonholonomic constraints," IEEE Transactions on Automatic Control, vol. 39, no. 3, pp. 609614,1994

[17] Z. G. Hou, A. M. Zou, L. Cheng, and M. Tan, "Adaptive control of an electrically driven nonholonomic mobile robot via backstepping and fuzzy approach," IEEE Transactions on Control Systems Technology, vol. 17, no. 4, pp. 803-815, 2009.

[18] R. Fierro and F. L. Lewis, "Control of a nonholonomic mobile robot using neural networks," IEEE Transactions on Neural Networks, vol. 9, no. 4, pp. 589-600, 1998.

[19] F. Hong, S. S. Ge, C. K. Pang, T. H. Lee, and Z. Sun, "Robust adaptive neuro-fuzzy control of uncertain nonholonomic systems," in Proceedings of the 8th IEEE International Conference on Control and Automation (ICCA '10), pp. 2201-2206, Xiamen, China, June 2010.

[20] M. Ye and L. X. Xiao, Analytical Mechanics, Tianjin University Press, Tianjing, China, 2001.

[21] W. Zhang, Modeling for Logic Systems of Stateflow, Xian Electronic and Science University Press, Xi'an, China, 2007. 


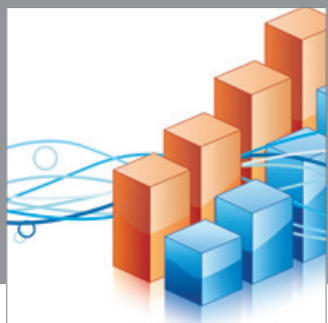

Advances in

Operations Research

mansans

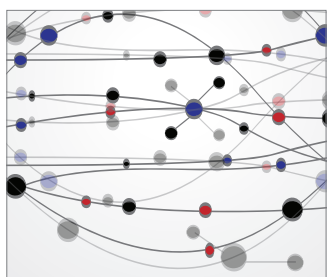

The Scientific World Journal
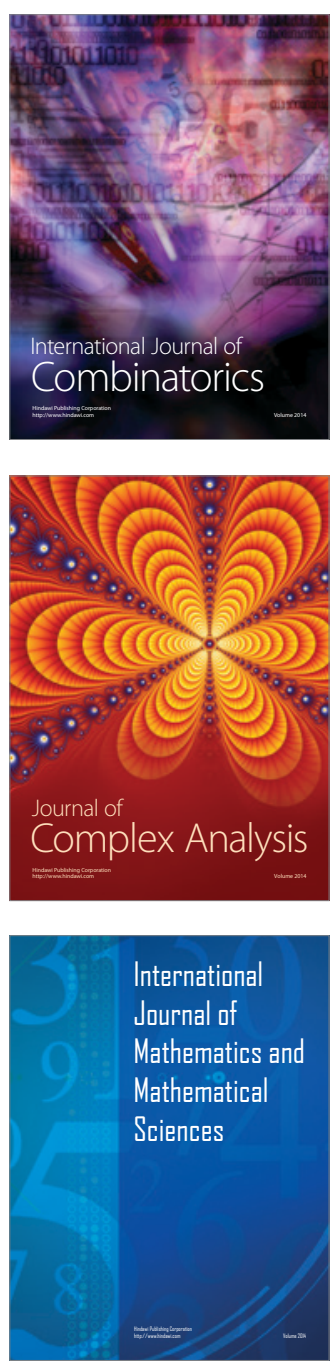
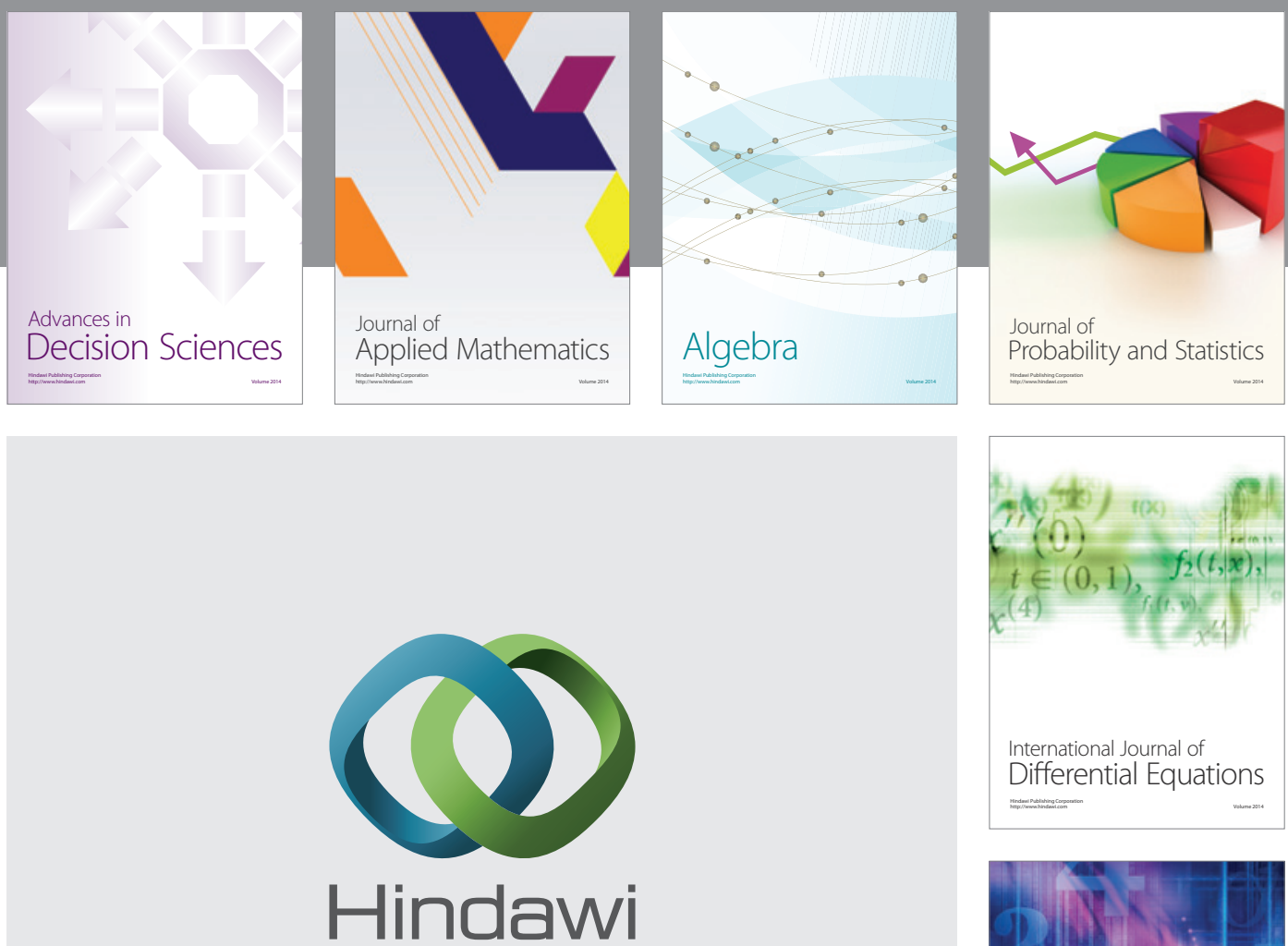

Submit your manuscripts at http://www.hindawi.com
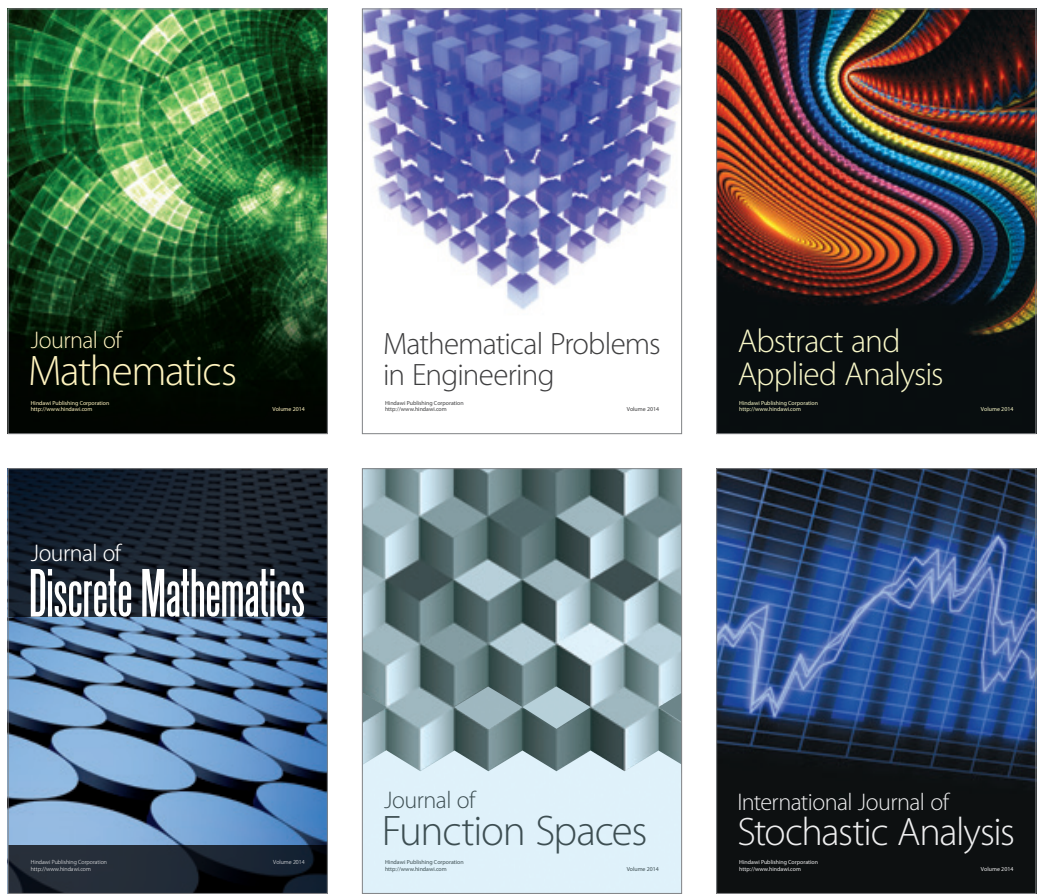

Journal of

Function Spaces

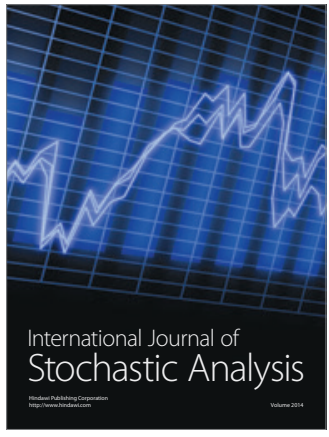

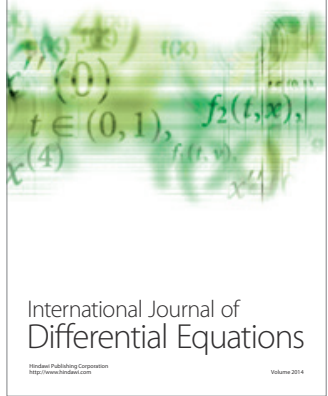
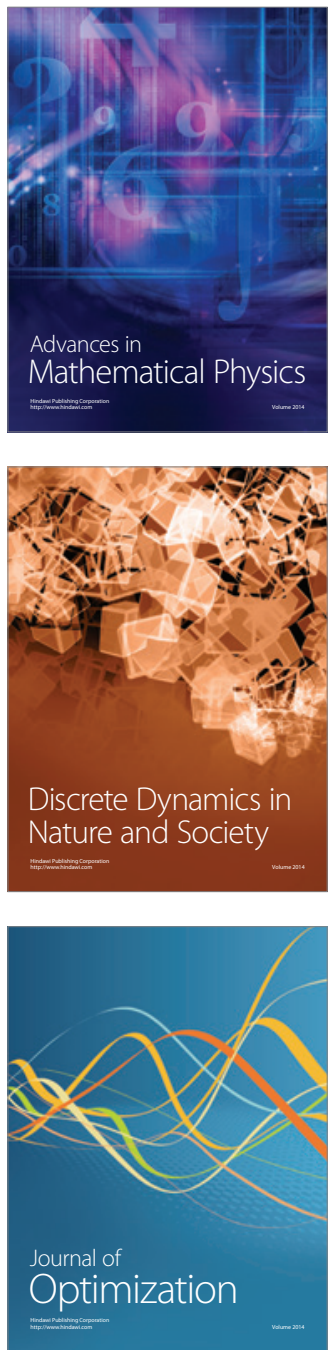